\title{
Potential measurement of the weak mixing angle with neutrino-electron scattering at low energy
}

\author{
Sanjib Kumar Agarwalla ${ }^{1}$ and Patrick Huber ${ }^{1}$ \\ ${ }^{1}$ Department of Physics, Virginia Tech, Blacksburg, VA 24060, USA
}

(Dated: November 2, 2018)

\begin{abstract}
We study the possibility to measure $\sin ^{2} \theta_{W}$ by neutrino-electron scattering at a value of the momentum transfer $Q \simeq 30 \mathrm{MeV}$ with a precision of $0.24 \%$, which is only a factor three below the one obtained by LEP-I at the $Z$-pole. The neutrino source is a proton beam dump providing a clean beam from muon decay at rest and the detector is a $100 \mathrm{kt}$ scale water Cerenkov detector, which results in about 20 million signal events.
\end{abstract}

PACS numbers: 12.15.-y, 12.15.Mm, 13.15.+g, 14.60.Lm, 14.60.-z

The Standard Model (SM) of particle physics provides a remarkably accurate description of a wide range of phenomena in nuclear and particle physics. The SM also unifies the weak and electromagnetic forces into one gauge group, $S U(2)_{L} \times U(1)_{Y}$. The electro-weak sector of the SM has been tested with utmost precision: in the weak sector of the theory precisions at $0.1 \%$ level are reached, whereas in the electromagnetic sector of the theory the precision is 1 part per billion or better. Despite its quantitative success, the SM has been shown to be incomplete by the discovery of neutrino mass, the existence of dark matter and the recent advent of dark energy. While particle accelerators, like the LHC, continue to look for new physics at ever higher energy scales, precision low energy observables have been and continue to be an invaluable tool to learn about the scale of new physics and to shed light into flavor sector, see e.g. [1]. Precise tests of the electro-weak sector of the SM are highly sensitive to the presence of oblique corrections affecting vacuum polarization of the photon, $Z$ and $W$ bosons through new particles in quantum loops and vertex corrections [2]. The weak mixing angle is defined by the ratio of the $\mathrm{SU}(2)_{L}$ gauge coupling $g$ and the $U(1)_{Y}$ gauge coupling $g^{\prime}$ by following relation $\sin ^{2} \theta_{W}=\frac{g^{\prime 2}}{g^{2}+g^{\prime 2}}$. It is one of the key parameters in this theory. Within the $\overline{M S}$ renormalization scheme this is a scale dependent quantity and thus its value is predicted to show a dependence on the energy scale at which it is measured. This renormalization group running of the weak mixing angle is an inevitable consequence of the electro-weak theory. Therefore, the experimental demonstration of the running of the weak mixing angle has been considered to be an experimentum crucis for the SM.

The first measurement of the weak mixing angle was described in [4], yielding $\sin ^{2} \theta_{W}=0.29 \pm 0.05$. Modern data on $\sin ^{2} \theta_{W}$ at low momentum transfer, $Q$, is based on experiments on atomic parity violation at $Q \sim 10^{-5} \mathrm{GeV}[5]$ and SLAC E158, a Møller scattering experiment at $Q=0.16 \mathrm{GeV}[6]$. Currently, the most precise measurement of $\sin ^{2} \theta_{W}$ was obtained from $e^{+} e^{-}$collisions at the $Z$-pole. Interestingly, the leptonic

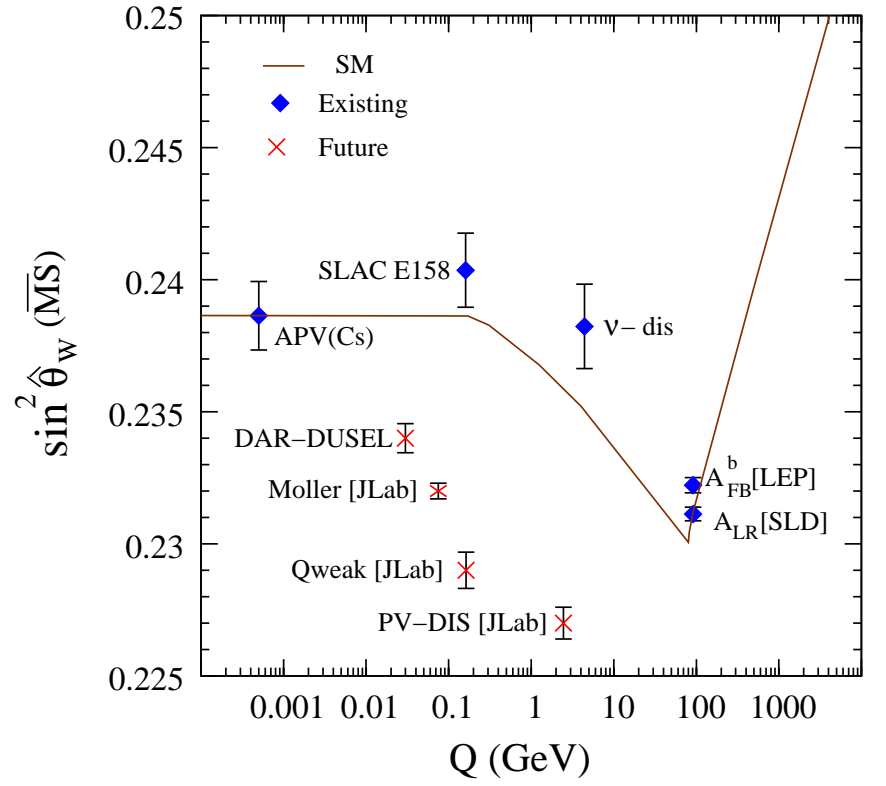

FIG. 1: A summary of the world data for the weak mixing angle as a function of the momentum transfer $Q$. The solid curve depicts the running of $\sin ^{2} \hat{\theta}_{W}$ in the $\overline{\mathrm{MS}}$ renormalization scheme [3]. The future experiments are shown with arbitrarily chosen vertical location. DAR-DUSEL denotes the result of this work.

$(0.23113 \pm 0.00021)$ and hadronic $(0.23222 \pm 0.00027)$ measurements of $\sin ^{2} \theta_{W}$ at $Z$-pole differ by 3.2 standard deviations [7]. The $\mathrm{NuTeV}$ collaboration reported a $3 \sigma$ deviation from the SM value of $\sin ^{2} \theta_{W}$ [8] using deep inelastic neutrino-nucleus scattering. Many clever explanations have been put forward to solve this puzzle [9]. These discrepancies could be a sign for new physics or maybe for not understood experimental effects. To clarify the origin of these discrepancies a set of new experiments to measure the weak mixing angle at various values of $Q$ has been proposed and approved, these are shown as red crosses in figure 1. There were several other proposals to measure the weak mixing angle, not all of them shown in figure 1, using conventional muon neutrino beams [10] at $Q \sim 0.1 \mathrm{GeV}$ or electron neutrinos 


\begin{tabular}{|c||c|c|c|}
\hline & $\nu_{e} e \rightarrow \nu_{e} e$ & $\nu_{\mu} e \rightarrow \nu_{\mu} e$ & $\bar{\nu}_{\mu} e \rightarrow \bar{\nu}_{\mu} e$ \\
\hline$\alpha$ & $\frac{1}{2}+\sin ^{2} \theta_{W}$ & $-\frac{1}{2}+\sin ^{2} \theta_{W}$ & $\sin ^{2} \theta_{W}$ \\
\hline$\beta$ & $\sin ^{2} \theta_{W}$ & $\sin ^{2} \theta_{W}$ & $-\frac{1}{2}+\sin ^{2} \theta_{W}$ \\
\hline
\end{tabular}

TABLE I: The values of $\alpha \& \beta$ in the SM for different processes involved in our case.

produced from muon decay at rest [1]. A 1 to $2 \%$ measurement of $\sin ^{2} \theta_{W}$ using a spallation neutron source was proposed in [12]. Another method was suggested in [13] to measure $\sin ^{2} \theta_{W}$ to about $1 \%$ at a reactorbased experiment. The possibility of measuring the weak mixing angle with a precision of $10 \%$ using low energy beta-beams was explored in [14]. At low $Q$, three new parity-violating electron scattering experiments namely Møller scattering 15], Qweak [16], and PVDIS [17] have been proposed at Jefferson Laboratory and would provide precision measurements of $\sin ^{2} \theta_{W}$ in the range 0.1 $-0.3 \%$ in the absence of physics beyond the SM.

Neutrino-electron scattering is a simple, purely leptonic weak interaction process that can play an essential role to prove the validity and perform precision tests of the SM [1, 18] as well as many of its extensions. Neutrino electron scattering is also quite sensitive to the weak mixing angle. In the SM, the tree-level ${ }^{1}$ differential cross section for neutrino-electron scattering can be written as 19]

$$
\frac{\mathrm{d} \sigma}{\mathrm{d} T}=\frac{2 G_{F}^{2} m_{e}}{\pi E_{\nu}^{2}}\left[\alpha^{2} E_{\nu}^{2}+\beta^{2}\left(E_{\nu}-T\right)^{2}-\alpha \beta m_{e} T\right],
$$

where $G_{F}$ is the Fermi constant, $m_{e}$ is the electron mass, $E_{\nu}$ is the incoming neutrino energy, $T$ is the electron recoil kinetic energy given by the kinematics and has the range $0 \leq T \leq T^{\max }=\frac{E_{\nu}}{1+m_{e} / 2 E_{\nu}}$. In the $\mathrm{SM}, \alpha$ and $\beta$ are process-dependent constants that depend on $\sin ^{2} \theta_{W}$ as given in Table I. The $\nu_{\mu}$-e cross section decreases with the increase in weak mixing angle while $\nu_{e}$-e and $\bar{\nu}_{\mu}$-e cross section increase. The recoil electron is produced at an angle $\theta$ to the incident neutrino direction where $\cos \theta=\left(1+m_{e} / E_{\nu}\right) / \sqrt{1+2 m_{e} / T}$. Thus the recoil electrons are strongly peaked along the neutrino direction if $T \gg m_{e}$. This forward peaking is used distinguish neutrino-electron scattering events from neutrino reactions on nuclei in the energy regime from a few $\mathrm{MeV}$ up to few tens of $\mathrm{MeV}$. In this energy range, neutrino-electron scattering can be observed experimentally in large water Cerenkov detectors with high efficiency and with nearly no background, as demonstrated by the results on solar neutrinos by Super-Kamiokande [20] and the Sudbury Neutrino Observatory [21].

\footnotetext{
${ }^{1}$ Higher order SM corrections are small and can be safely neglected.
}

Here we suggest an experiment to measure $\sin ^{2} \theta_{W}$ up to a precision of $0.24 \%$ based on neutrinos from pion and anti-muon decay at rest (DAR). These anti-muons are obtained from the decay of stopped $\pi^{+}$; the $\pi^{+}$in turn are produced in a proton beam dump. The novel concept compared to earlier proposals is that we will use the energy dependence and not the total rate, $c f$. [11], of the recoil electron spectrum which is possible due to the much higher statistics.

Technologically, the key are high-intensity, low energy proton sources, which currently are developed for various applications and have been previously suggested as driver for a stopped pion neutrino source [22]. The electrons from neutrino electron scattering will be observed in a large-size, $\mathcal{O}(100 \mathrm{kt})$, water Cerenkov detector like the one proposed for the Deep Underground Science and Engineering Laboratory (DUSEL) 23, 24].

We assume the same proton source parameters as in [22]: $100 \mu \mathrm{s}$ pulse every $500 \mu \mathrm{s}, 2 \mathrm{GeV}$ protons and $9.4 \times 10^{22}$ protons on target per year, which results in $4 \times 10^{22}$ neutrinos per flavor and year. These numbers apply for one of these sources, throughout of this paper we assume 2 of them at the same location. The running time is 5 years, thus the total exposure is $9.4 \times 10^{23}$ protons on target. The resulting neutrino flux is shown in the inset of figure 2. The flavor composition and spectrum are well understood from first principles. Due to the strong $\pi^{-}$absorption in the target, the $\bar{\nu}_{e}$ contamination in the beam is very tiny $\left(\sim 10^{-4}\right)$ and we therefore will neglect it. For a review on stopped pion neutrino sources and the associated physics, see [25].

We assume a $300 \mathrm{kt}$ water Cerenkov detector consisting of two volumes of right cylinder of $150 \mathrm{kt}$ each, separated by a distance of $60 \mathrm{~m}$. The neutrino source is in the middle between the two detector modules so that both the detector volumes will receive the same amount of neutrino flux. Accounting for the geometric acceptance, we find an average distance to target corresponding to the actual distance between the source and the center of mass of the detectors, which is $54 \mathrm{~m}$.

The incoming $\nu_{e}, \nu_{\mu}$ and $\bar{\nu}_{\mu}$ will scatter with the electrons inside the detector and we will measure the kinetic energy and the direction of the recoil electron. We assume $75 \%$ detection efficiency, $\epsilon$ 20]. The energy resolution, $\delta T$, for the electron is $5 \% \sqrt{\mathrm{E} / \mathrm{GeV}}$ which is a conservative choice compared to Super-Kamiokande II [20]. This corresponds $\delta T=8.7 \mathrm{MeV}$ at $T=30 \mathrm{MeV}$. We work with a bin size of $5 \mathrm{MeV}$ for $T_{A}$. We have checked that even if we consider a bin size of $10-20 \mathrm{MeV}$ the results will not change appreciably. We consider a reconstructed energy threshold of $10 \mathrm{MeV}$. Note, that the electron performance of a such detectors can be calibrated to a very high level of precision using a small electron linear accelerator [26], in particular the energy scale can be determined to better than $1 \%$.

The scattered electrons from the various neutrino fla- 

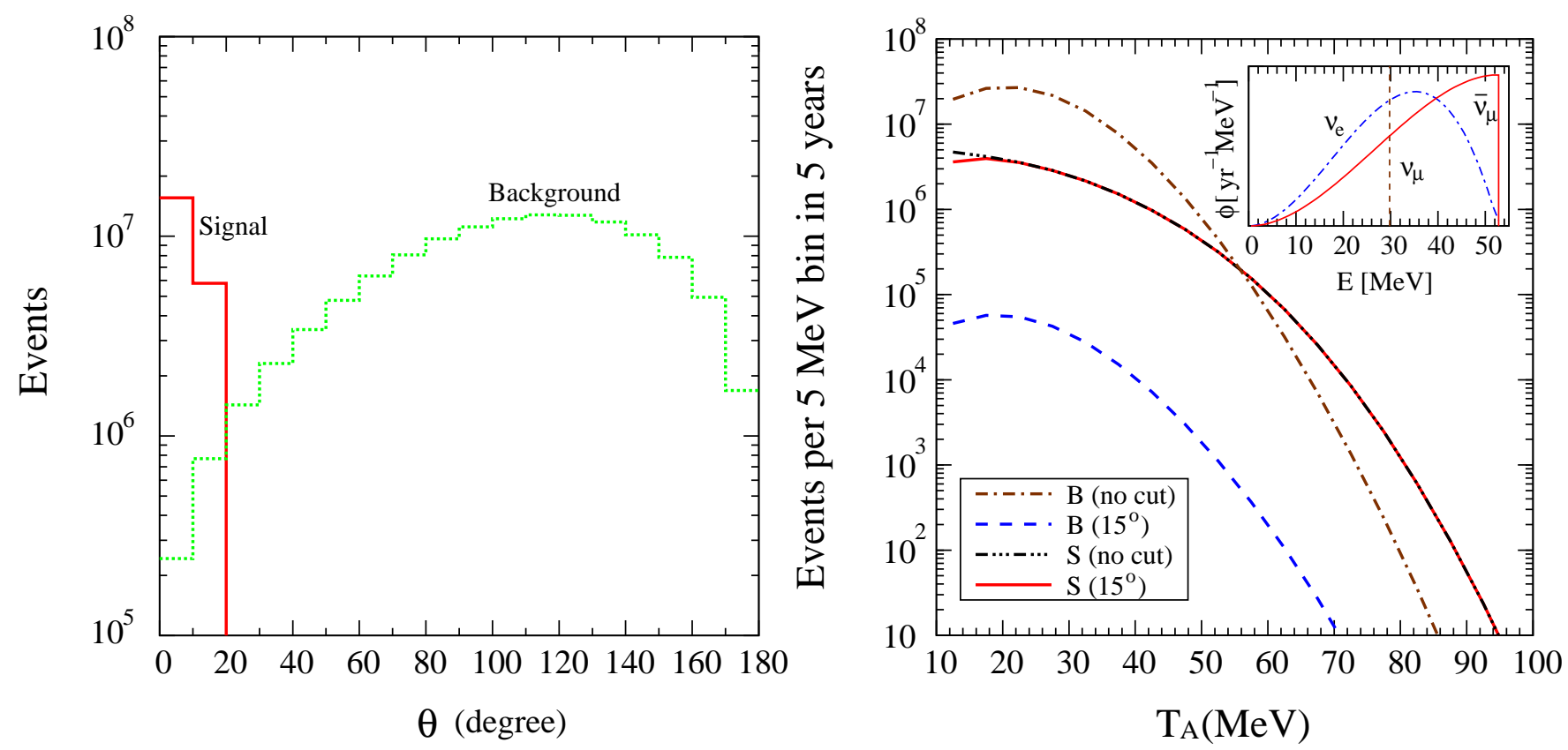

FIG. 2: The left hand panel shows the angular distribution of the signal and background events. The red solid line is the signal and the green dotted line indicates the background. The right hand panel shows the neutrino-electron scattering events in five years with two cyclotrons as a function of reconstructed recoil electron kinetic energy, $T_{A}$. The expected background events from charged-current $\nu_{e}$-Oxygen reaction are also shown. The impact of angular cut on signal and background events are also depicted. In the inset, the energy distribution of neutrinos in a DAR beam is shown.

vors of a DAR source are not distinguishable and their contributions must be incoherently added to calculate the sensitivity of $\sin ^{2} \theta_{W}$. The number of neutrino-electron elastic scattering events in the $i$-th bin of the reconstructed recoil electron kinetic energy, $T_{A}$ is given by

$$
\begin{aligned}
\mathrm{N}_{i}= & \frac{c t n_{e} \epsilon}{4 \pi}\left\langle\frac{1}{L^{2}}\right\rangle \sum_{\text {flavors }}^{\nu=\nu_{\mu}, \nu_{e}, \bar{\nu}_{\mu}} \int_{0}^{E_{\nu}^{\max }} \mathrm{d} E_{\nu} \int_{0}^{T^{\max }} d T \\
& \int_{T_{A_{i}}^{\min }}^{T_{A_{i}}^{\max }} \mathrm{d} T_{A} R\left(T, T_{A}\right) \phi_{\nu}\left(E_{\nu}\right) \frac{\mathrm{d} \sigma_{\nu}\left(E_{\nu}, T\right)}{\mathrm{d} T}
\end{aligned}
$$

where $c$ is the number of accelerators, $t$ is total running time, $n_{e}$ is the number of target electrons in the detector, $\epsilon$ is the detector efficiency and $R\left(T, T_{A}\right)$ is the Gaussian energy resolution function of the detector. $\left\langle 1 / L^{2}\right\rangle$ is the average inverse square distance of the detector from the source. $\phi_{\nu}\left(E_{\nu}\right)$ is the incoming neutrino flux and $E_{\nu}^{\max }=$ $m_{\mu} / 2$ is the maximum available energy of the neutrinos from a DAR source, with $m_{\mu}$ being the muon mass. The resulting event distribution as a function of $T_{A}$ is shown in figure 2 as solid line.

The by far dominant source of beam-on background are electrons produced from charged-current reactions on oxygen, $\nu_{e}+\mathrm{O} \rightarrow e^{-}+\mathrm{F}[28]$. The angular distribution of these background events is strongly backward peaked whereas the neutrino-electron scattering signal events are sharply forward peaked, as shown in the left hand panel of figure 2, where the shape and size of these backgrounds

\begin{tabular}{||c||c||c||c||c||}
\hline \hline$\theta$ & S & B & S/B & $\begin{array}{c}\text { rel. error } \\
\text { on } \sin ^{2} \theta_{W}\end{array}$ \\
\hline no cut & $21.2 \times 10^{6}$ & $122 \times 10^{6}$ & 0.17 & $0.57 \%$ \\
\hline $30^{\circ}$ & $21.2 \times 10^{6}$ & $1.4 \times 10^{6}$ & 15 & $0.25 \%$ \\
\hline $15^{\circ}$ & $19.8 \times 10^{6}$ & $0.26 \times 10^{6}$ & 78 & $0.24 \%$ \\
\hline \hline
\end{tabular}

TABLE II: The expected number of signal and background events with and without angular cut have been given in second and third column respectively. The relative, $1 \sigma$, error in measuring $\sin ^{2} \theta_{W}$ is quoted in the last column.

is taken from Ref. [28]. By applying suitable angular cuts, we can increase the signal over background ratio 11 , 28]. The angular resolution of a water Cerenkov detectors averaged over the energy range considered here is better than $15^{\circ}$ [26]. Beam-off backgrounds are negligible in our case due to the deep underground location 22]. The effect of the angular cut on the background is shown in the right hand panel of figure 2 , the dash dotted line is the background without any cuts, whereas the dashed lined is the background which remains with a $15^{\circ}$ cut. As can be seen from table III even a $30^{\circ}$ cut would be sufficient. The importance of a tight angular cut is to eliminate the need to know the angular distribution of the background to a high level of precision.

To illustrate the effect, we are trying to measure, we 


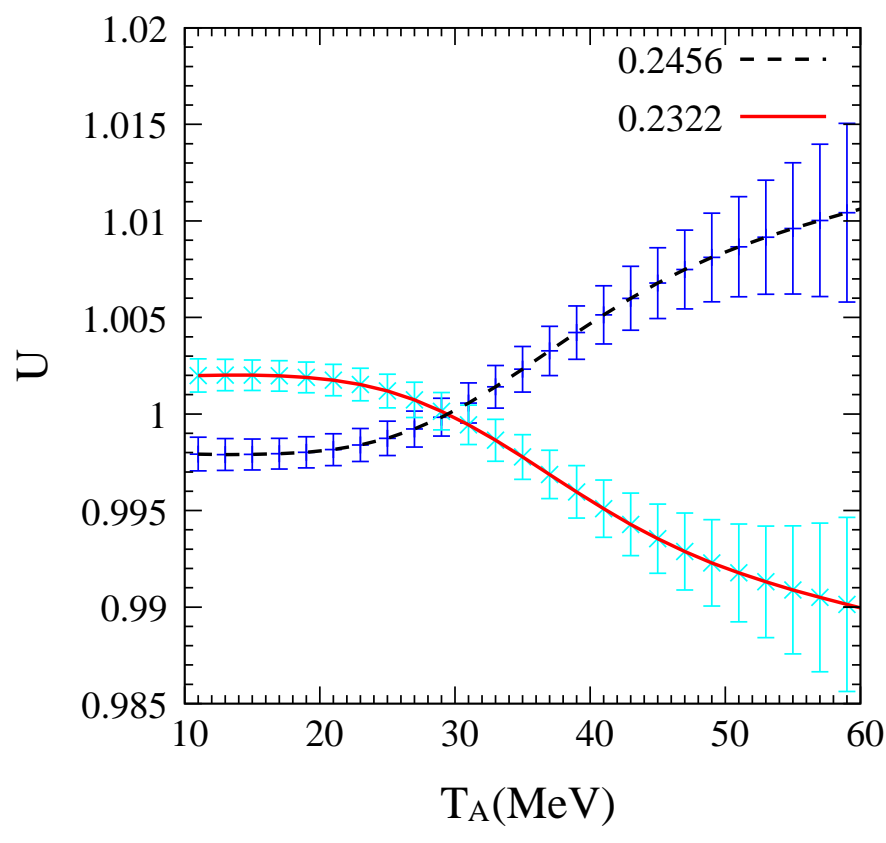

FIG. 3: The variation of $U$ (as defined in the text) with $T_{A}$. The bin width is $2 \mathrm{MeV}$ and the error bars are statistical $1 \sigma$ errors. The two lines are for different values of $\sin ^{2} \theta_{W}$ as indicated in the legend.

define a shape parameter $U$

$$
U=\frac{N_{i}\left(\sin ^{2} \theta_{W}\right)}{\hat{N}_{i}\left(\sin ^{2} \hat{\theta}_{W}\right)} \frac{\sum_{i=1}^{n} \hat{N}_{i}\left(\sin ^{2} \hat{\theta}_{W}\right)}{\sum_{i=1}^{n} N_{i}\left(\sin ^{2} \theta_{W}\right)} .
$$

where $N_{i}$ is the number of recoil electrons of the reconstructed-energy bin $i$ as a function of the fitted value of $\theta_{W}$, whereas $\hat{N}_{i}$ is the number of events predicted for a given, true value of $\theta_{W}=\hat{\theta}_{W}$. $U$ obviously is invariant under a rescaling of the total rate, therefore it contains shape information only and $U \equiv 1$ for all energies if $\theta_{W}=\hat{\theta}_{W}$. From the energy dependence of $U$, as shown in figure 3 . we can extract the value of $\sin ^{2} \theta_{W}$. The lines are for two different values of $\sin ^{2} \theta_{W}$. In our analysis, we have taken the true value of weak mixing angle $\sin ^{2} \hat{\theta}_{W}=0.23863$, which corresponds to the value measured at the Z-pole [27] evolved down to $Q=0.03 \mathrm{GeV}$ using the $\overline{M S}$ scheme. The energy dependence of $U$ on $T_{A}$ is due to the superposition of the recoils from the mono-energetic $\nu_{\mu}$ line from $\pi$-decay and the continuous spectra resulting from $\mu$-decay. This is also the reason for the inflection point at $30 \mathrm{MeV}$, which is the position of the $\nu_{\mu}$ line. Since we are relying on the energy dependence of our signal in order to suppress the impact of systematical errors, we show in table III to what extent our results change if the the energy resolution of the detector is varied. The precision on the weak mixing angle deteriorates by a factor of two for a decrease in resolution by a factor of three. This in turn translates into a requirement for sufficient photo cathode coverage similar to the one of Super-K.

\begin{tabular}{||c||c||}
\hline \hline $\begin{array}{c}\delta T \\
(\times \sqrt{\mathrm{E} / \mathrm{GeV}})\end{array}$ & $\begin{array}{c}\text { rel. error on } \sin ^{2} \theta_{W} \\
(1 \sigma)\end{array}$ \\
\hline $5 \%$ & $0.25 \%$ \\
\hline $7.5 \%$ & $0.29 \%$ \\
\hline $10 \%$ & $0.34 \%$ \\
\hline $12.5 \%$ & $0.4 \%$ \\
\hline $15 \%$ & $0.48 \%$ \\
\hline \hline
\end{tabular}

TABLE III: The relative $1 \sigma$ error in the measurement of $\sin ^{2} \theta_{W}$ as a function of the energy resolution of the detector.

For our statistical analysis we use the so called pull approach as used in [29]. We bin our data in $T_{A}$ into bins of $5 \mathrm{MeV}$ and do not use $U$ but the event rates. We include a $5 \%$ systematic on the total number of signal events and a (uncorrelated) $5 \%$ systematic on the total number of background events. Note, that systematics twice as large would not change our results appreciably, demonstrating that this is a shape based measurements.

The expected number of signal and background events with and without angular cut have been given the in second and third column of table II, respectively. The final precision of our set-up in measuring $\sin ^{2} \theta_{W}$ is quoted in the last column. We can see an improvement by factor of two when we impose an angular cut of $30^{\circ}$ on $\theta$ which helps us to get rid of the background by a substantial amount. If we were to perform a rate-only measurement instead, the precision in measuring $\sin ^{2} \theta_{W}$ deteriorates to $2.8 \%$, i.e. by one order of magnitude. The use of the shape information is very effective in suppressing the impact of systematics and thus, the question arises whether the measurement is statistics limit with our current choice of beam luminosity and detector size. Therefore, we study the impact of changing the exposure over two orders of magnitude; the result thereof is shown in figure 4. The error on the weak mixing angle scales as $1 / \sqrt{\mathcal{L}}$, where $\mathcal{L}$ is the exposure in protons on target. This supports the conclusion that this measurement is indeed statistics limited over a wide range of exposures. Figure 4 also allows to extrapolate the attainable precision for different source-detector configurations. Note, that when the detector mass (and hence the detector geoemetry) or source-detector distance changes, the geometrical acceptance has to be reevaluated, however in most circumstances the resulting correction is expected to be relatively minor.

To summarize, the use of shape information, which we propose here for the first time in this context, is the key to a high precision measurement of $\sin ^{2} \theta_{W}$ using neutrino-electron scattering. The use of shape information is only possible if a sufficiently large event sample can be obtained. To this end the combination of highintensity proton sources with very large detectors is cru- 


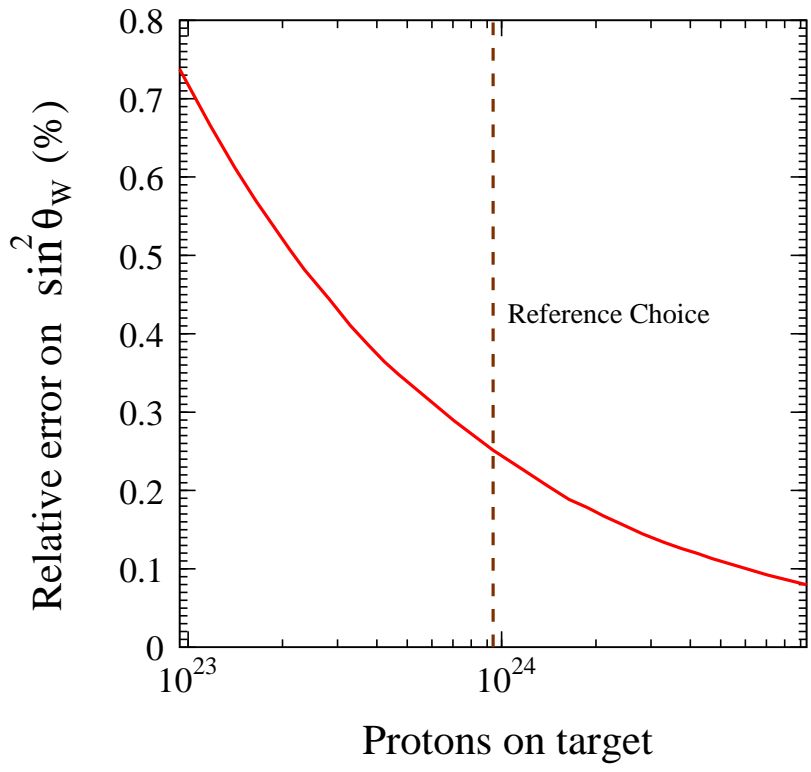

FIG. 4: The relative $1 \sigma$ error on $\sin ^{2} \theta_{W}$ in per cent as a function of exposure. The dotted vertical line shows our reference choice for the exposure.

cial. This configuration can be a natural part of the proposed physics program for DUSEL. Our proposed experiment will provide $\mathrm{a} \simeq 0.24 \%$ measurement of $\sin ^{2} \theta_{W}$ comparable to the currently most precise results.

We would like to thank J.M. Link, M. Pitt, R. Raghavan, K. Schollberg, and, T. Takeuchi for useful discussions. This work has been in part supported by the U.S. Department of Energy under award number de-sc0003915.

[1] J. Erler and M. J. Ramsey-Musolf, Prog. Part. Nucl. Phys. 54, 351 (2005).

[2] M. E. Peskin and T. Takeuchi, Phys. Rev. Lett. 65, 964 (1990); Phys. Rev. D 46, 381 (1992); G. Altarelli and R. Barbieri, Phys. Lett. B 253, 161 (1991); G. Degrassi, A. Sirlin and W. J. Marciano, Phys. Rev. D 39, 287 (1989).
[3] J. Erler and M. J. Ramsey-Musolf, Phys. Rev. D 72, 073003 (2005).

[4] H. S. Gurr, F. Reines and H. W. Sobel, Phys. Rev. Lett. 28, 1406 (1972).

[5] C. S. Wood et al., Science 275, 1759 (1997); S. G. Porsev, K. Beloy and A. Derevianko, Phys. Rev. Lett. 102, 181601 (2009).

[6] P. L. Anthony et al. [SLAC E158 Collaboration], Phys. Rev. Lett. 92, 181602 (2004); Phys. Rev. Lett. 95, 081601 (2005).

[7] The ALEPH, DELPHI, L3, OPAL, SLD Collaborations, Phys. Rept. 427, 257 (2006).

[8] G. P. Zeller et al. [NuTeV Collaboration], Phys. Rev. Lett. 88, 091802 (2002) [Erratum-ibid. 90, 239902 (2003)].

[9] S. Davidson et al., JHEP 0202, 037 (2002); W. Loinaz et al., Phys. Rev. D 67, 073012 (2003).

[10] L. A. Ahrens et al., Phys. Rev. D 41, 3297 (1990); P. Vilain et al. [CHARM-II Collaboration], Phys. Lett. B 335, 246 (1994).

[11] L. B. Auerbach et al. [LSND Collaboration], Phys. Rev. D 63, 112001 (2001).

[12] R. Imlay and G. J. VanDalen, J. Phys. G 29, 2647 (2003).

[13] J. M. Conrad et al., Phys. Rev. D 71, 073013 (2005).

[14] A. B. Balantekin, J. H. de Jesus and C. Volpe, Phys. Lett. B 634, 180 (2006).

[15] K. S. Kumar, AIP Conf. Proc. 1182, 660 (2009).

[16] M. L. Pitt [Qweak Collaboration], AIP Conf. Proc. 1182, 706 (2009).

[17] P. Souder, Prepared for 16th International Workshop on Deep Inelastic Scattering and Related Subjects (DIS 2008), London, England, 7-11 Apr 2008.

[18] W. J. Marciano and Z. Parsa, J. Phys. G 29, 2629 (2003).

[19] P. Vogel and J. Engel, Phys. Rev. D 39, 3378 (1989).

[20] J. P. Cravens et al. [Super-Kamiokande Collaboration], Phys. Rev. D 78, 032002 (2008).

[21] B. Aharmim et al. [SNO Collaboration], Phys. Rev. C 72, 055502 (2005).

[22] J. M. Conrad and M. H. Shaevitz, Phys. Rev. Lett. 104, 141802 (2010).

[23] http://www.lbl.gov/nsd/homestake/

[24] S. Raby et al., arXiv:0810.4551 [hep-ph].

[25] R. Lazauskas and C. Volpe, arXiv:1004.0310 [hep-ph].

[26] M. Nakahata et al. [Super-Kamiokande Collaboration], Nucl. Instrum. Meth. A 421, 113 (1999).

[27] W. M. Yao et al. [Particle Data Group], J. Phys. G 33, 1 (2006).

[28] W. C. Haxton, Phys. Rev. C 37, 2660 (1988).

[29] P. Huber, M. Lindner and W. Winter, Nucl. Phys. B 645, 3 (2002). 